Meta

Journal des traducteurs

Translators' Journal

\title{
Collocations dans une base de données terminologique et lexicale
}

\section{Ulrich Heid et Gerhard Freibott}

Volume 36, numéro 1, mars 1991

La terminologie dans le monde : orientations et recherches

URI : https://id.erudit.org/iderudit/002882ar

Aller au sommaire du numéro

Éditeur(s)

Les Presses de l'Université de Montréal

ISSN

0026-0452 (imprimé)

Découvrir la revue

Citer cet article

Heid, U. \& Freibott, G. (1991). Collocations dans une base de données terminologique et lexicale. Meta, 36(1), 77-91. d'utilisation que vous pouvez consulter en ligne. 


\title{
COLLOCATIONS DANS UNE BASE DE DONNÉES TERMINOLOGIQUE ET LEXICALE
}

\author{
Ulrich HeId et Gerhard Freibott \\ Université de Stuttgart, KRUPP Industrietechnik $\mathrm{GmbH}$, \\ Stuttgart et Duisburg, RFA
}

\section{INTRODUCTION}

Le service de traduction et de documentation de l'entreprise KRUPP Industrietechnik GmbH (Duisburg) a décidé, en 1989, de construire, sur la base d'expériences faites avec différents produits utilisés depuis des années, une base de données lexicographique et terminologique qui vienne remplacer ou compléter les outils alors en usage. La base de données que nous avons conçue se distingue de bon nombre de produits commercialisés ou en développement; parmi les traits distinctifs, il faut noter avant tout :

- l'interfaçage possible avec d'autres composantes d'un poste de travail informatisé pour traducteurs ou auteurs techniques, telles que traitement de textes, aides à la comparaison automatique de différentes versions de textes, archives de textes, etc;

- la possibilité de combiner la base de données avec des bases de connaissances externes; elle peut être combinée avec la version informatisée d'un catalogue de pièces de rechange structuré, comme les bases de connaissances des sytèmes experts, par des relations IS-A et PART-OF;

- la multifonctionnalité linguistique : la base de données a été conçue pour être utilisable, même en dehors du domaine strictement normalisé de la langue technique hautement «terminologisée», aussi bien comme dictionnaire de thème que comme dictionnaire de version' ${ }^{1}$ a à cela s'ajoute la possibilité, utile avant tout aux auteurs techniques, d'utiliser la base de données comme un dictionnaire monolingue ${ }^{2}$

C'est surtout ce dernier aspect de la multifonctionnalité linguistique qui a contribué au développement d'une description lexicale et terminologique différente de nombre de bases de données existantes. Pour servir des usagers dont les exigences étaient assez diverses, il fallait repenser le modèle désormais «classique» des bases de données terminologiques, tel qu'il est aux fondements, pour n'en citer qu'un exemple, d'EURODICAUTOM ${ }^{3}$.

Du côté linguistique, l'application des concepts qui se sont dessinés à travers les discussions menées parmi les lexicographes, dans les cinq à dix dernières années, est la caratéristique la plus importante de la base de données. Bien que nous sachions, grâce à ces discussions, assez exactement quels sont les problèmes que pose la multifonctionnalité dictionnairique, peu d'essais ont été faits, à notre connaissance, pour mettre ces résultats à profit dans la conception et l'implantation d'un outil lexical et terminologique pour traducteurs et auteurs techniques.

Deux aspects de cette discussion lexicographique et terminographique des années 80 nous ont paru d'un intérêt particulier, à savoir

- le traitement cohérent des relations d'équivalence;

- le traitement des collocations. 
Dans le présent article, nous nous concentrerons sur le traitement des collocations dans notre base de données. Nous exposerons d'abord les problèmes que posent les collocations aux traducteurs et aux auteurs de textes techniques; dans le chapitre suivant, nous donnerons un aperçu des approches descriptives proposées en lexicographie et en terminographie. Sur la base de ces éléments descriptifs, nous pourrons exposer la méthode suivie aussi bien dans la description que dans la présentation du phénomène dans notre base de données.

\section{LE PROBLÈME}

\subsection{SURVOL}

Le but du traducteur, aussi bien que de l'auteur technique, est de fournir un texte non seulement cohérent au niveau terminologique, mais aussi «lisible» sur le plan stylistique. Tant qu'il traduit d'une langue étrangère vers sa langue maternelle ou qu'il produit des textes en langue maternelle, les outils en place lui permettent d'atteindre la cohérence terminologique souhaitée, et il est censé être à même de pourvoir lui-même à la lisibilité, grâce à sa maîtrise de la langue.

Les problèmes liés aux caractéristiques linguistiques des collocations peuvent faire obstacle, par contre, à ce que le traducteur ou l'auteur arrive à la même «lisibilité» dans la langue étrangère; et il faut admettre que la production de textes en langue étrangère est loin, du moins dans l'industrie allemande, d'être quantitativement négligeable.

L'un des éléments principaux de la «lisibilité» d'un texte, en langue générale ou technique, est l'apparition de collocations, c'est-à-dire de combinaisons de lexèmes préférées par une communauté linguistique. Or, les collocations, comme créer un fichier, annuler une demande en informatique, stimuler, accentuer, accélérer une reprise, une inflation rampante, l'oscillation des indices dans le langage économique et boursier, doivent être apprises avec la langue étrangère, comme les termes. D'une part, les traducteurs et les auteurs techniques doivent donc disposer d'un «trésor» collocationnel important pour atteindre le degré nécessaire de lisibilité dans la langue cible, d'autre part, les caractéristiques linguistiques des collocations interdisent, dans la plupart des cas, une simple traduction littérale des constructions de la langue de départ. Le traitement que reçoivent les collocations dans les dictionnaires techniques ne facilite pas la tâche du traducteur, car la plupart des dictionnaires sont bel et bien pauvres en collocations. S'il en figure dans les bases de données, il n'est pas chose aisée d'accéder à l'information nécessaire: nous reviendrons sur ces points plus tard.

Dans le reste de ce chapitre, nous essaierons d'exposer les caractéristiques linguistiques des collocations; nous nous appuyons sur les travaux de (Hausmann 1979, 1985,1988 ) qui nous semblent tout à fait applicables, en dehors de la langue générale, considérée par Hausmann, aussi à la langue technique.

Un résumé des travaux de Hausmann du point de vue lexicographique a été publié par Benson (1989), auteur de l'un des rares dictionnaires de collocations (de la langue standard anglaise 5 ).

\subsection{CARACTÉRISTIQUES LINGUISTIQUES DE LA COLLOCATION : LE CÔTÉ DESCRIPTIF}

2.2.1. Les collocations dans la description monolingue

Avec Hausmann (1985), nous entendons par collocation une combinaison polaire non arbitraire de deux lexèmes qui a un caractère conventionnel à l'intérieur d'un groupe linguistique. 
Nous parlons de combinaisons de lexèmes, ce qui exclut par exemple les combinaisons de noms ou de verbes avec des prépositions et ce qui réduit la liste typologique sur laquelle nous nous basons, aux quatre types suivants:

- Substantif - Verbe:

* rééquilibrer les marchés [ÉCONOMIE]

* annuler une demande [INFORMATIQUE: LOGICIELS]

* téléscoper la flèche (d'une grue) [ENGINS DE LEVAGE]

- Substantif - Adjectif

* ressources renouvelables [AGRICULTURE]

* élution graduée [CHROMATOGRAPHIE]

- Adjectif - Adverbe

* éperdument amoureux

- Verbe - Adverbe

* désirer qqc vivement

Nous parlons de combinaisons «polaires», pour souligner le fait que l'un des deux lexèmes détermine ceux avec lesquels il peut être combiné. Dans le langage de l'informatique, on peut dire créer un fichier, mais non pas ??établir un fichier ou ?? concevoir un fichier.

Cet aspect polaire peut aussi être décrit comme étant une propriété sémantique et lexicale : pour exprimer un contenu donné qui sert à modifier ou déterminer un lexème $\mathrm{A}$, le choix entre différents lexèmes $\mathrm{B}_{1}, \mathrm{~B}_{2}, \ldots, \mathrm{B}_{\mathrm{n}}$ est conditionné par le choix de $\mathrm{A}^{6}$.

L'auteur qui choisit fichier, dans l'exemple donné plus haut, n'a plus un choix illimité entre des verbes quasi synonymes comme concevoir, faire, créer, établir, ouvrir, pour dire qu'il fait en sorte que le fichier vienne en existence.

En nous servant de la terminologie utilisée par Hausmann (1985), nous distinguons à l'intérieur de la collocation entre la base et le collocateur; la base est le lexème (A) qui détermine quels autres lexèmes $\left(B_{1}, B_{2}, \ldots, B_{n}\right.$, les collocateurs) peuvent se combiner avec lui.

Nous parlons de caractère conventionnel pour souligner que les collocations sont lexicalisées et considérées par les locuteurs natifs comme étant des «produits semi-finis» de leur langue (Hausmann). C'est aussi ce qui distingue les collocations d'autres combinaisons de lexèmes qui sont arbitraires, c'est-à-dire non lexicalisées et le produit «du hasard du texte».

Les collocations ont un caractère conventionnel à l'intérieur d'une communauté linguistique; c'est aussi vrai pour les «microcommunautés linguistiques» que sont les entreprises, les groupes professionnels, etc: comme on peut observer des différences terminologiques d'entreprise à entreprise, ou entre le langage des ouvriers et celui des ingénieurs ou du marketing, les collocations peuvent aussi varier selon le groupe, le registre, etc.

2.2.2. Les collocations dans la description contrastive

Les équivalents de collocations ne sont pas prédictibles dans la traduction. Cela signifie que

- l'équivalent d'une collocation ne doit pas forcément être une collocation;

- si l'équivalent d'une collocation est aussi une collocation, seule la «base» (Hausmann) se traduit indépendamment de la collocation, mais l'équivalence des collocateurs ne peut être décrite que dans le contexte de la collocation. Cela découle du fait que les bases choisissent les collocateurs avec lesquels ils se combinent. 
Nous illustrerons le deuxième point par des exemples pris de la langue standard aussi bien que de la langue technique; dans l'illustration 1, nous reproduisons une partie de l'article s.v. dresser du Dictionnaire du français contemporain (DFC); dans l'illustration 2, nous reprenons quelques-unes des collocations françaises décrites dans le $\mathrm{DFC}$, avec à côté leurs équivalents allemands et des collocateurs impossibles en allemand.

1. dresser [drese] v. tr. $1^{\circ}$ Dresser quelque chose. le mettre debout, le mettre dans une position verticale ou voisine de la verticale : Dresser in mât (syn. : PLANTER). Dresser une échelle contre le mur. On a dressé une barrière (syn. : ÉLEVER). Ils araient dressé leur tente (syn. : MONTER). Dresser un monument, une statue (syn. : ÉRIGER). Dresser la tête, le buste (syn. : LEVER). $-2^{\circ}$ Dresser quelque chose, l'établir, le mettre par écrit : Dresser un hilun, une liste, un plan, un constat, un procès-verhal. $-3^{\circ}$ Dresser la table, le couvert, disposer les couverts pour un repas (syn. : METTRE). $-4^{\circ}$ Dresser un piège, le préparer (syn. : TENDRE). - 5" Dresser une personne contre une autre, la mettre en opposition avec elle, l'exciter contre elle. $6^{\circ}$ Faire dresser les cheveux sur la tête à quelqu'un, lui causer de la frayeur, lui inspirer une grande horreur. II Dresser l'oreille, devenir soudain attentif à ce qui se dit.

\begin{tabular}{|ll|lll|}
\hline Français & Allemand & & \\
\hline Collocateur & Base & Base & Collocateur & $\begin{array}{l}\text { Collocateur } \\
\text { impossible }\end{array}$ \\
\hline dresser & $\begin{array}{l}\text { des barricades } \\
\text { un budget } \\
\text { une } \\
\text { contravention } \\
\text { un bilan }\end{array}$ & $\begin{array}{l}\text { Barikaden } \\
\text { Budget }\end{array}$ & $\begin{array}{l}\text { errichten } \\
\text { erstellen }\end{array}$ & (*errichten $)$ \\
& Strafzettel & ausstellen & $(*$ (*erstellen) \\
& Bilanz & aufstellen & $(*$ ausstellen $)$ \\
\hline
\end{tabular}


Le même phénomène se rencontre dans la langue spécialisée.

Ce sont surtout les collocations composées de noms et de verbes qui posent problème: le verbe allemand abschalten couvre une gamme de dénotations exprimées dans des collocations plus spécifiques en français. Dans le manuel d'une autogrue produite par l'entreprise, nous avons trouvé les exemples suivants de collocations avec les traductions respectives:

\begin{tabular}{|l|l|}
\hline Allemand & Français \\
\hline den Wandler abschalten & débrancher le convertisseur \\
das Ventil abschalten & couper la soupape \\
die Differentiale abschalten & déverrouiller les différentiels \\
die Kranbewegung abschalten & arrêtuer la manœuvre de la grue \\
das Teleskopieren abschalten & interrompre le télescopage \\
\hline
\end{tabular}

\subsection{LES COLLOCATIONS DANS UN DICTIONNAIRE BILINGUE OU MULTILINGUE:} LE CÔTÉ DE LA PRÉSENTATION

Après avoir exposé les caractéristiques linguistiques des collocations, il faut revenir sur les besoins des traducteurs et auteurs techniques en matière de présentation lexicographique et terminographique des collocations.

Le présent paragraphe sera donc consacré aux conclusions qu'il faut tirer, pour la présentation (surtout contrastive) de collocations des caractéristiques linguistiques mentionnées.

Les collocations ne sont pas des combinaisons arbitraires. Beaucoup d'exemples linguistiques donnés dans les dictionnaires ou les bases de données à titre illustratif le sont. Alors, il faut explicitement indiquer quels exemples sont arbitraires et quelle partie du matériau illustratif donné dans une entrée de dictionnaire contient des collocations. Or une telle distinction fait souvent défaut, et dans la plupart des bases de données terminologiques, les collocations se trouvent - quasiment introuvables - parmi les exemples.

Les collocations sont polaires. Il faut distinguer entre la base et le collocateur. Hausmann (1988) a montré que cette distinction a un impact sur l'endroit dans un dictionnaire où l'on cherche une collocation.

Selon lui, l'auteur qui écrit un texte sait de quel concept il veut parler, mais il cherche un épithète ou un verbe ou, dans le cas de verbes ou d'adjectifs, un adverbe qui peut venir modifier, décrire ou compléter le concept en question. L'auteur d'un texte accédera alors au dictionnaire par l'entrée d'une base, cherchant un collocateur convenable. Selon Hausmann, la même situation se produit dans la traduction vers une langue étrangère, c'est-à-dire dans le dictionnaire de thème.

Si l'utilisateur d'un dictionnaire bilingue se trouve dans une situation de version, cependant, il n'est pas a priori clair par où il entrera dans le dictionnaire. Étant donné qu'il a devant lui un texte en langue étrangère qu'il doit décoder, il n'est pas certain qu'il se rende compte d'être en présence d'une collocation. Il n'y a donc pas moyen d'établir un système non redondant de recherche, car l'utilisateur ne peut pas se reporter à l'entrée 
de la base d'une collocation là où il ne s'aperçoit pas du caractère collocationnel d'une combinaison de lexèmes. L'usager du dictionnaire de version espère donc pouvoir trouver la collocation aussi bien dans l'entrée du collocateur que dans celle de la base.

Étant donné que notre base de données sera utilisée dans les deux activités de thème et de version, ainsi que dans la production de textes, les collocations doivent être accessibles à partir de leurs deux éléments, indifféremment.

Toute collocation ne doit pas forcément se traduire par une collocation; il faut aussi prévoir des cas où une collocation a un équivalent qui est un seul lexème. Dans la conception d'une base de données, il faut tenir compte de cette éventualité. Si l'on traite les collocations en sous-entrées, on court le risque de produire des entrées partiellement cycliques, ce qui n'est pas souhaitable.

\subsection{RÉSUMÉ PROVISOIRE: EXIGENCES VIS-À-VIS D'UNE MODÉLISATION DE COLLOCATIONS DANS UNE BASE DE DONNÉES}

Sur la base des exigences générales formulées pour la construction de notre base de données, ainsi qu'en fonction des caractéristiques linguistiques et des problèmes d'accès aux entrées, les exigences suivantes ont guidé la modélisation du phénomène de la collocation dans notre base de données :

- Les collocations ont toutes les caractéristiques de lexèmes: comme celles qui consistent en une seule chaîne de caractères, elles peuvent par exemple être porteurs de marques de variation; elles peuvent avoir aussi bien des équivalents collocationnels que des équivalents «en un mot».

Alors elles auront le même statut de «lemme» ${ }^{7}$ que les termes.

- L'utilisation de la base de données en tant que dictionnaire de thème aussi bien qu'en tant que dictionnaire de version nécessite que l'usager puisse accéder à la collocation et à ses équivalents tant dans l'article de la base que dans celui du collocateur.

Alors les entrées des collocations seront automatiquement reliées par des pointeurs explicites avec les entrées des bases et celles des collocateurs.

- Il faut séparer les collocations des phrases illustratives (et des locutions idiomatiques).

Alors, aux entrées séparées des collocations correspondra un attribut particulier dans les entrées des bases et des collocateurs. Les collocations ne seront pas mélangées avec les matériaux illustratifs.

\section{LES COLLOCATIONS DANS LA RECHERCHE TERMINOLOGIQUE ET LEXICOGRAPHIQUE}

La description des collocations a fait l'objet de discussions entre linguistes depuis que J.R. Firth a utilisé le terme, dans les années 50. À côté d'une tradition contextualiste britannique, les lexicographes, et parmi eux, surtout ceux qui travaillent sur la lexicographie bilingue, tels que Hausmann et Kromann, se sont occupés du sujet. Ce n'est que depuis quelques années que les terminographes se sont intéressés à la matière, tandis que les manuels de terminologie qui suivent la tradition de Wüster ne tiennent pas explicitement compte de ce phénomène. En linguistique, la combinatoire lexicale a été décrite dans des essais théoriques aussi bien que dans des fragments dictionnairiques, par l'équipe d'Igor A. Mel'čuk.

\subsection{APPROCHES LINGUISTIQUES}

Ici, nous n'essaierons pas de résumer les travaux du contextualisme britannique qui furent en quelque sorte à la base de la discussion linguistique sur les collocations. Les travaux de Firth (1951) et de Halliday $(1961,1966)$ ont été résumés en allemand par Roos 
(1975) et dans l'histoire du contextualisme de Steiner (1983). Un aperçu très utile et compact, publié en anglais, est l'article de Van Roey (1978) qui peut aussi servir comme introduction au sujet.

$\mathrm{Au}$ début, les contextualistes ne cherchaient ni à définir une approche lexicographique descriptive ou présentationnelle, ni à fournir une théorie de la combinatoire; les premières expériences ont été consacrées, par contre, à la description du style d'auteurs littéraires.

Les développements récents des recherches contextualistes sur les collocations sont résumées dans Sinclair (1987), un rapport issu des activités du projet dictionnairique COBUILD. L'approche contextualiste s'est alors vue combiner avec des procédures lexicostatistiques basées sur l'analyse de corpus (cf. aussi les travaux de Bergenholtz (1981)

À l'occasion de la parution du BBI Combinatory Dictionary of English, les auteurs de cet ouvrage ont décrit dans différents articles les choix qu'ils avaient faits dans leur dictionnaire (cf. Benson $1986 \mathrm{a}, \mathrm{b}, \mathrm{c}$ ).

Indépendamment, Hausmann (1977) a analysé quelques-uns des dictionnaires d'épithètes, tels que Bar (1930) et Lacroix (1931) monolingues, ainsi que Reum/Chambille (1911), un dictionnaire «de style à l'usage des Allemands».

Dans Hausmann (1979), le premier aperçu d'une méthodologie pour un dictionnaire collocationnel est donné; un essai plus détaillé avec une description linguistique plus fine se trouve dans Hausmann (1985), ainsi que, sous l'angle des implications pour la lexicographie bilingue, dans Hausmann (1988). Ce n'est que dans Benson (1989) que les travaux de Hausmann se sont vus discutés extensivement dans le monde anglophone; Kromann (1989) a repris les idées de Hausmann et les a interprétées sur le fond de sa théorie des dictionnaires bilingues.

La description des propriétés linguistiques des collocations ainsi que la discussion de leurs implications pour la présentation lexicographique et terminographique repose sur ces travaux.

Plus ou moins parallèlement, les germanistes lexicographes et lexicologues autour de Helbig et Viehweger (cf. Viehweger 1982, Brausse 1987) ont approché le problème du côté d'une description valencielle des verbes et adjectifs.

Dans le cadre de ces approches, la description de collocations a surtout été motivée par l'aspect didactique de la lexicographie, c'est-à-dire les dictionnaires pour étrangers, dictionnaires d'apprentissage, mais aussi par la méthodologie de l'apprentissage lexical, etc. (cf. à ce sujet aussi les travaux de Cowie 1978, 1981).

Moins dans un souci pédagogique que dans le cadre d'une théorie sémantique et syntaxique, le groupe de Mel'čuk a analysé le phénomène de la combinatoire lexicale. La description des collocations a été systématisée grâce à l'introduction des «fonctions lexicales» qui servent à décrire un sens abstrait combiné avec un lexème dans une collocation. Selon Mel'čuk et al. (1984), l'application à des lexèmes d'opérateurs abstraits relativement généraux (tels que «un haut degré de», «se passer», «faire en sorte que... se produise», etc.) peut se réaliser dans différentes lexicalisations (collocationnelles), selon les lexèmes auxquels s'appliquent les opérateurs.

Cette conception est en un sens assez proche de celle de Hausmann, et elle a l'avantage de se prêter à une formalisation: le modèle de l'application d'un opérateur à un élément lexical donné permet d'exprimer la distinction entre base et collocateur: les éléments (mots clés, dans la terminologie de Mel'cuk, bases selon Hausmann) auxquelles s'appliquent les opérateurs choisissent les lexèmes avec lesquels ils se combinent. L'équipe de Mel'cuk a décrit des fragments lexicaux du russe et du français (cf. Mel'čuk et al. 1984, 1989), aussi bien que d'autres langues; des propositions visant à la 
formalisation (Mel'cuk/Polguère 1987) et à l'exploitation des descriptions lexicales dans des systèmes de traitement automatique du language (cf. Kittredge/Polguère/Iordanskaja 1988, Polguère 1990) ont été faites.

\subsection{APPROCHES TERMINOLOGIQUES ET TERMINOGRAPHIQUES}

Les sources classiques de la terminologie ne tiennent pas compte de la collocation; Wüster ne fait pas mention de la combinatoire lexicale, et les manuels du début des années 80, tels que Arntz/Picht (1982) en Allemagne, ne sont pas très riches non plus.

Les terminologues se sont rendu compte, cependant, surtout au cours des cinq dernières années, qu'il est utile d'intégrer une composante textuelle et combinatoire dans les descriptions terminographiques: les travaux des groupes de Picht et de Kromann ont particulièrement contribué à cette prise de conscience. Picht (1987 a/b), ainsi que les travaux antérieurs de 1983 et 1985 (en danois) ont mis en évidence la fonction terminologique des verbes; en même temps, les terminographes et linguistes qui ont analysé des dictionnaires spécialisés se sont aperçus de l'absence d'entrées autres que nominales et du manque d'information contextuelle (cf. par exemple le compte rendu de Wagner 1986, de Rossenbeck 1989, etc.). Ces deux types de travaux semblent avoir massivement contribué à la naissance de la «phraséologie des langues de spécialité», domaine de recherche en apparition, constaté et encouragé par Cormier (1989). Entre temps, aussi bien en France qu'en Autriche, des tables rondes ont été organisées, dont le but était de faire le bilan des recherches en phraséologie spécialisée entreprises ces dernières années.

D'autre part, les lexicographes et les terminographes ont continué leurs échanges (cf. par exemple DANLEX-group 1987), et de nouvelles propositions pour l'analyse de systèmes terminologiques, tenant compte de phénomènes combinatoires (cf. Heltai 1989) ont été faites. Elles ont été accompagnées par des propositions visant à la restructuration de dictionnaires spécialisés, allant jusqu'à l'introduction des dispositifs descriptifs et présentationnels de Mel'cuk (cf. Frawley 1988).

Dans le domaine des dictionnaires spécialisés, quelques nouveautés sont intervenues: Cohen (1986) est un «lexique de cooccurrents» qui répertorie des collocations de plus d'une centaine de substantifs de la langue française de la bourse, selon un double classement: selon les phases d'un procès (début, croissance, déclin, fin), et selon les catégories des collocateurs (nom, verbe, adjectif).

Selon l'expérience rapportée par Knowles (1988) et par Tomaszczyk (1989), beaucoup de dictionnaires spécialisés du russe contiennent de l'information collocationnelle. La recherche phraséologique et contextuelle est aussi assez développée en Union Soviétique.

\subsection{LES COLLOCATIONS DANS LES BASES DE DONNÉES TERMINOLOGIQUES}

À notre connaissance, les bases de données terminologiques ne contiennent pas de dispositifs particuliers pour le traitement de collocations.

Les collocations figurent la plupart du temps parmi le matériau illustratif. Rarement, elles sont explicitement distinguées des exemples non collocatifs. Qui plus est, l'utilisateur a souvent du mal à accéder à une collocation qui se trouve parmi les exemples donnés à titre d'illustration d'un terme donné. Dans certaines bases de données, il est impossible de chercher à travers toutes les informations (à l'aide d'un browser). Ceci est possible dans une base de données telle qu'EURODICAUTOM qui supporte la recherche à travers tous les types d'informations.

Dans les bases de données à étendue moins large, les collocations font souvent complètement défaut. Si, techniquement, il est possible de retenir des entrées composées 
de plusieurs mots, il est rare que l'utilisateur puisse, avec un certain confort, accéder à ces entrées à partir de l'une de leurs composantes.

Les auteurs des bases de données ne se sont jusqu'à présent, semble-t-il, pas occupés de la représentation de collocations dans les bases de données lexicales ou terminologiques. Même des modèles avancés tels que celui discuté dans Mayer/Maier (1987) ne tiennent pas compte de ce type d'information.

Ce peu d'attention portée au problème de la «phraséologie des langues de spécialité» dans les outils disponibles pourrait s'expliquer par le fait que les terminographes viennent seulement de prendre conscience du problème et que, dans la conception de bases de données, souvent peu de questions «purement» linguistiques ont été prises en compte. Un autre problème non négligeable réside dans la convivialité des outils. Beaucoup d'utilisateurs sont très réticents vis-à-vis de l'idée d'avoir davantage de fonctions d'une base de données à maîtriser qu'une demi-douzaine de commandes d'accès: pour éviter que l'outil perde son caractère attrayant à cause d'une certaine surcharge, beaucoup d'auteurs se limitent à l'essentiel, c'est-à-dire à l'équivalence de termes.

\section{VERS UNE MODÉLISATION DU PHÉNOMENE DE LA COLLOCATION DANS UNE BASE DE DONNÉES}

Dans la conception et l'implantation de notre base de données, nous avons essayé de tenir compte des caractéristiques linguistiques des collocations autant que possible.

Dans le paragraphe présent, nous décrivons la structuration générale de la base de données, avant de donner des détails au sujet du traitement des collocations. Ensuite, nous montrerons, à l'aide de reproductions de l'écran, quelles options existent pour l'accès à l'information collocationnelle.

\subsection{LES INFORMATIONS DÉCRITES DANS LA BASE DE DONNÉES}

Nous donnerons d'abord un aperçu général (abrégé) des types d'informations qui peuvent être décrites dans la base de données ${ }^{8}$.

Nous distinguons entre différents types d'objets linguistiques et conceptuels qui peuvent être décrits dans la base de données. À un niveau plus abstrait, cette classification fait évidemment une distinction entre concepts et lexèmes.

Étant donné qu'il est souhaitable que différents utilisateurs puissent créer leur terminologie à partir de différentes langues, considérées à chaque fois comme étant la langue source dans une relation de traduction, les lexèmes des différentes langues ont aussi un statut d'objets séparés. Ceci implique pour la modélisation (dans le format d'une base de données relationnelle) qu'un concept, un lexème français et un lexème allemand ou anglais sont tous traités comme des objets séparés; les relations de synonymie, d'équivalence mais aussi de «lexicalisation», c'est-à-dire le rapport entre un concept et un lexème, sont alors des relations entre objets de la base de données relationnelle.

Les concepts se distinguent des lexèmes par le type d'attributs et de relations qu'ils supportent.

Les informations données au sujet des concepts sont les suivantes:

- rapport avec un numéro d'identification qui renvoie à un objet concret répertorié dans un catalogue de pièces de rechange informatisé;

- rapport avec les illustrations (images projetables sur l'écran) contenues dans le catalogue

de pièces de rechange;

- structuration des concepts par les relations IS-A et PART-OF. 
Les informations données au sujet des lexèmes peuvent être subdivisées en deux types; nous avons cru bon de représenter certaines informations par des attributs, d'autres par des relations entre lexèmes.

Les informations décrites par des attributs de lexèmes sont encore subdivisées dans trois «paquets»:

- description documentaire et statistique :

* terminologue(s) qui a/ont travaillé sur l'entrée en question, avec date de la première entrée et de la dernière mise à jour;

- description terminologique:

* définitions;

* citations, exemples illustratifs, bibliographie;

- description linguistique :

* catégorie (nom, verbe, adjectif);

* attributs morphosyntaxiques (genre, nombre, propriétés syntaxiques, etc.);

* marquage concernant la variation linguistique (registre, langage d'entreprise, etc.).

Nous représentons par des relations les propriétés linguistiques suivantes:

- les relations entre lexèmes d'une langue:

* synonymie et antonymie;

* les abréviations;

- les collocations;

- les relations entre lexèmes de différentes langues:

* l'équivalence, la quasi-équivalence et la relation de paraphrase ${ }^{9}$.

Ce tableau correspond assez bien au modèle structuraliste de la description lexicale: les propriétés paradigmatiques des unités lexicales sont représentées par des relations, tandis que les propriétés syntagmatiques et les «marques d'usage» sont modélisées par des attributs.

\subsection{LES COLLOCATIONS DANS LA BASE DE DONNÉES}

Dans le chapitre 2.4., nous avons résumé les exigences formulées vis-à-vis de la description de collocations dans notre base de données; ces exigences reposent, d'une part sur la multifonctionnalité envisagée de la base de données, d'autre part sur les propriétés linguistiques des collocations. Le chapitre présent sera consacré à une description de la modélisation réalisée dans la base de données et sera organisé parallèlement au chapitre 2.4 .

4.2.1. Les collocations traitées comme «lemmes» de la base de données

Plus haut, nous avons indiqué que les concepts aussi bien que les lexèmes sont traités en tant qu'objets de la base de données. Les bases de données relationnelles se prêtent assez bien à une modélisation qui regarde les lexèmes comme étant des objets linguistiques qui possèdent des propriétés (décrites par des attributs) et qui entretiennent des relations de divers types avec d'autres objets linguistiques.

Étant donné que les collocations peuvent être décrites comme ayant en grande partie les mêmes types de propriétés que les lexèmes consistant «en un mot», nous pouvons leur donner le même statut d'objet linguistique qu'aux lexèmes «ordinaires».

Parmi les propriétés linguistiques qui peuvent être définies aussi bien pour des lexèmes «ordinaires» que pour des collocations, citons à titre d'exemple

- dans la description terminologique

* citations, exemples illustratifs;

- dans la description linguistique :

* le marquage concernant la variation linguistique (registre, langage d'entreprise, etc.). 
De même, il est possible et nécessaire de décrire les relations dont les collocations sont un élément, à savoir surtout les synonymes et les équivalents de collocations données.

Il est évident, d'autre part, que les attributs catégoriels et morphosyntaxiques ne peuvent pas avoir la même signification, lorsqu'ils sont utilisés dans la description de collocations.

Une description catégorielle des collocations est utile seulement si elle tient compte de la catégorie des deux lexèmes impliqués. Nous nous servons de la classification illustrée dans 2.2.1. qui distingue quatre types de combinaisons de lexèmes, à savoir

- substantif - verbe

- substantif — adjectif

- adjectif - adverbe

- verbe - adverbe.

Cette classification est grossière, il est vrai, mais en vue d'une utilisation de la base de données qui ne nécessite pas trop de formation de la part de l'utilisateur, nous avons décidé de nous limiter à une telle typologie. Il est évident que la base de données permettrait l'introduction de sous-classes plus spécifiques, et l'implantation subdivise comme le fait Cohen (1986) dans son dictionnaire - les collocations substantif - verbe en deux types, selon que le nom est un «sujet» ou un autre complément du verbe.

Notons que cette sous-classification des collocations en types «catégoriels» a un avantage supplémentaire: lorsque le terminologue fait entrer des données dans la base, il est guidé par des menus différents, selon la langue et le type catégoriel de la collocation traitée: une telle situation d'entrée de données uniformisée permet d'assurer un haut degré de cohérence.

\subsubsection{Assurer l'accessibilité des collocations}

Le fait que les collocations soient traitées au même titre que les lexèmes simples permet de les relier avec d'autres objets linguistiques sans besoin d'introduire de nouvelles relations; le rapport avec des synonymes, des équivalents, etc. est donc établi par les relations habituelles, à partir des «entrées» des collocations.

Or, l'utilisateur est susceptible de rencontrer deux types de situations: la situation de version, dans laquelle il risque d'hésiter sur le statut collocationnel d'une combinaison de lexèmes rencontrée dans un texte en langue étrangère; ou bien la situation de thème (ou de production de textes) dans laquelle il cherche un collocateur d'une certaine catégorie pour une base donnée, dans un texte qu'il veut produire.

Il est donc nécessaire que la base de données donne accès aux collocations à partir de leurs deux éléments et que l'entrée d'une collocation renvoie aussi aux entrées de ses composantes.

Pour tenir compte de ces exigences, nous avons établi un système de double pointage, permettant de relier automatiquement les entrées respectives. Les renvois sont créés lorsque le terminologue fait entrer des collocations dans la base de données; il est obligé de construire une entrée explicite pour chaque collocation. $̇$ partir de la classe collocationnelle (définie en termes de catégories des deux lexèmes impliqués, p. ex. N A pour nom - adjectif), le système infère les catégories des deux lexèmes et vérifie leur présence dans la base de données. Ensuite, elle introduit dans chacune des entrées des deux éléments de la collocation un pointeur qui la relie avec l'entrée de la collocation.

Si plusieurs entrées existent qui ont le même «lemme» et la même catégorie (homographes), le terminologue est invité à décider quel homonyme sera considéré comme apparaissant dans la collocation. 
Si une seule entrée est trouvée, le terminologue aura à décider s'il veut relier la collocation avec cette entrée ou s'il préfère créer une entrée homographe qui sera reliée avec la collocation.

En l'absence d'entrées convenables, une entrée «minimale» est créée automatiquement qui ne contient que le lemme, la catégorie et un renvoi à la collocation en question.

La présentation des renvois dépend de la catégorie des entrées. Nous renvoyons d'une base à tous les collocateurs dont les combinaisons avec la base en question sont décrites dans le dictionnaire électronique; pareillement, les entrées de collocateurs contiennent des indications quant aux bases avec lesquelles ils apparaissent (dans le fragment décrit dans la base de données). Pour faciliter la recherche, nous avons introduit, dans les entrées des bases et des collocateurs, des listes de lemmes avec lesquels les lexèmes respectifs se combinent. Étant donné que les verbes et les adjectifs peuvent aussi bien être base que collocateur, plusieurs listes sont accessibles dans les entrées de ces catégories, à savoir:

- les noms dont ils sont les collocateurs;

- les adverbes dont ils sont les bases.

Les lemmes répertoriés dans ces listes apparaissent en ordre alphabétique.

Il faut souligner que la création des renvois est automatique et hors de l'influence du terminologue; celui-ci peut évidemment ôter des collocations à la base de données, ce qui entraîne la suppression des relations respectives.

\subsubsection{Collocation vs. matériau illustratif}

La description des dispositifs utilisés dans la modélisation et l'implantation de la composante collocationnelle de notre base de données aura mis en évidence que les collocations sont entièrement séparées des exemples illustratifs des lexèmes. Elles peuvent, par contre, être illustrées elles-mêmes par des exemples contextuels. Les entrées des lexèmes dont elles sont composées contiennent des renvois aux collocations aussi bien que du matériau illustratif non collocationnel, séparément.

\section{L'ACCÈS À L'INFORMATION COLLOCATIONNELLE}

Dans ce qui suit, nous essayons d'illustrer l'application de la base de données en suivant de près un exemple d'utilisation.

Dans la traduction d'un texte français en allemand, la collocation desserrer le frein (d'un véhicule) doit être traduite. Un traducteur qui se rend compte du fait que desserrer le frein est une collocation peut alors chercher cette collocation telle quelle dans la base de données. Elle contiendra une entrée collocationnelle pour desserrer le frein et l'utilisateur est informé de l'existence d'équivalents. Il les voit sur l'écran en actionnant la touche F6. Deux équivalents allemands sont décrits dans la base de données, présentés en tant que synonymes: (die) Bremse lösen ou bien (die) Bremse öffnen.

On peut aussi imaginer une situation dans laquelle le traducteur ne connait pas l'équivalent d'un collocateur, mais où il n'est pas certain du statut collocationnel de l'unité qu'il doit traduire. Il cherche alors dans la base de données à partir du collocateur qui lui pose problème. Prenons une situation de traduction vers le français: le traducteur a rencontré le verbe allemand lösen et il hésite entre desserrer, relâcher, etc. Alors il cherche lösen. La base de données indique que le verbe apparaît dans des collocations avec les noms suivants: (die) Bremse lösen, (die) Federblockierung lösen, etc. L'utilisateur peut alors choisir la collocation dont il a besoin, par exemple Federblockierung lösen; l'équivalent dans ce cas est déverrouiller le blocage de suspension. 
Dans l'utilisation de la base de données par des auteurs techniques, les collocations sont souvent appelées à partir de leurs bases. Un auteur qui veut décrire les freins du véhicule et les actions de ralentissement et de freinage est intéressé par la possibilité de voir les collocations disponibles avec la base frein. Alors il cherche frein et appelle la liste de collocations, où frein est l'objet d'un verbe, comme dans serrer le frein, desserrer le frein, relâcher le frein. Étant donné que chaque collocation peut être décrite selon le modèle des autres lemmes, par exemple sur le plan des marques d'usage, l'auteur a accès à l'information nécessaire pour décider quelle collocation il utilisera.

\section{CONCLUSION}

Le but des travaux dont les résultats ont été décrits dans la présente contribution était de développer une base de données terminologique et lexicale qui ait une certaine utilité dans la production de textes aussi bien que dans la traduction de et vers une langue étrangère.

Ces exigences nous semblent répondre à deux tendances actuelles: d'une part la tendance de la pratique d'utiliser un même outil dans la traduction et la rédaction techniques, activités ayant tendance à se rejoindre au point de s'amalgamer; d'autre part la tendance de la recherche terminologique de tenir compte, dans une mesure croissante, des aspects textuels et stylistiques des documents techniques, et, de pair, de développer la «phraséologie des langues de spécialité», discipline encore jeune mais en plein essor et susceptible de profiter des résultats de la lexicographie.

Dans notre article, nous avons décrit les problèmes que posent les collocations, aux niveaux descriptif et présentationnel, dans la construction d'un dictionnaire interrogeable en interactif; nous avons tracé les grandes lignes des approches antérieures, en nous concentrant sur celle de Hausmann (1985, 1988), avant de montrer l'application à la construction d'une base de données.

Faute d'expérience avec un nombre significatif d'utilisateurs, nous ne pouvons pas vraiment donner d'estimations sur la convivialité de la base de données, mais dans sa réalisation, il fallait trouver un compromis entre le degré de détail requis par la description linguistique et terminologique et la convivialité et la simplicité de l'utilisation. Une évaluation de ce compromis reste à faire; elle sera possible lorsque davantage d'utilisateurs se seront servis du système.

\section{NOTES}

1. La différence entre les deux types de dictionnaires bilingues a été décrite par Al (1983) et par Kromann/Riiber/Rosbach (1984, 1989); cf. plus bas.

2. Nous avons décrit les caractéristiques aussi bien linguistiques qu'informatiques de la base de données dans Freibott/Heid (1990a).

3. Pour une description comparative des grandes bases de données terminologiques, cf. Hvalkof (1985).

4. Une description de notre approche se trouve dans Freibott/Heid (1990b).

5. Cf. Benson/Benson/lison (1986 a/b).

6. Cf. la description de «combinatoire lexicale» dans Mel'cuk et al. $(1984,1989)$ qui, dans l'essentiel, nous semble être tout à fait parallèle avec celle de Hausmann

7. Ces collocations sont done aussi considérées comme objets de la base de données par où on peut entrer dans le dictionnaire.

8. Nous nous bornerons à une description assez générale. Les détails ont été discutés dans Freibott/Heid (1990 a).

9. Nous distinguons entre ces trois différents «degrés» d'équivalence, $\mathrm{cf}$. Freibott/Heid (1990 b). 


\section{BIBLIOGRAPHIE}

ACRICOLA, Erhard (Leitung); BRAUSSE, Ursula; KARL, Ilse; LUDWIG, Klaus-Dieter and Dieter VIEHWEGER (1987): Linguistische Studien; Reihe A Arbeitsberichte, Berlin, 169/1.

ACRICOLA, Erhard (Leitung), BRAUSSE, Ursula, KARL, Ilse and Klaus-Dieter LUDWIG (1987): Linguistische Studien; Reihe A Arbeitsberichte, Berlin, 169/2.

AL, Bernard P. F. (1983): «Dictionnaire de thème et dictionnaire de version», Revue de phonétique appliquée, 66-68, pp. 201-211.

BAR, Elvire D. (1930): Dictionnaire des épithètes et qualificatifs, Paris, Garnier.

BAZELL, C.E.; CATFORD, J.C. and R. H. ROBINS (Eds.)(1966): In Memory of J.R.Firth, London.

BENSON, Morton, BENSON, Evelyn and Robert ILSON (1986): The Lexicographic Description of English. (Studies in Language Companion Series, 14), Amsterdam/Philadelphia, Benjamins.

BENSON, Morton, BENSON, Evelyn and Robert ILSON (1986): The BBI Combinatory Dictionary of English. A Guide to Word Combinations, Amsterdam/ Philadelphia, Benjamins.

BENSON, Morton (1989): "The Structure of the Collocational Dictionary", International Journal of Lexicography, 2.

BERGENHOLTZ, Henning and Joachim MUGDAN (Eds.): Lexikographie und Grammatik. Akten des Essener Kolloquiums zur Grammatik im Wörterbuch, Lexicographica, Series Maior, 3.

BRAUSSE, Ursula (1987): "Kollokations - und Valenzrelationen," Agricola, 2, pp. 269-323.

COHEN, Betty (1986): Lexique de cooccurrents, Montréal, Linguatech.

CORMIER, Monique C. : «La terminologie : du terme au texte», de Schaetzen, pp. 212-218.

COWIE, Anthony P. (1978): "The Place of Illustrative Material and Collocations in the Design of a Learner's Dictionary,"Strevens, Peter (Ed.): In Honour of A.S. Hornby, Oxford, pp. 127-139.

CZAP, Hans and Christian GALINSKI (1987) (Eds.): Terminology and Knowledge Engineering, Frankfurt/M., Indeks.

THE DANLEX-GROUP (1987): Descriptive Tools for Electronic Processing of Dictionary Data, Tübingen, Max Niemeyer.

FIRTH, John Rupert (1957): "Modes of Meaning," Papers in Linguistics 1934-1951, London, pp. 190-215.

FRAWLEY, William (1988): "New Forms of Specialized Dictionaries", International Journal of Lexicography $I-3$, pp. $189-213$.

FREIBOTT, Gerhard and Ulrich HEID (1990): "Terminological and Lexical Knowledge for Computer-Aided Translation and Technical Writing", Stuttgart/Duisburg, internal paper submitted to the Second International Congress on Terminology and Knowledge Engineering, TKE'90, Trier.

FREIBOTT, Gerhard, Ulrich HEID (1990): "Zur Darstellung von Äquivalenten in einer terminologischlexikalischen Datenbank für Übersetzer und technische Autoren", à paraître dans: Actes de GLDVJahrestagung 1990, Siegen.

HALLIDAY, Michael A.K. (1961): "Categories of the Theory of Grammar," Word. Journal of the Linguistic Circle of New York, 17, pp. 241-292.

HALLIDAY, Michael A.K. (1966): "Lexis as a linguistic level,"

Bazell/Catford/Robins (Eds.), pp. 148-162.

HAUSMANN, Franz-Josef (1977): Einführung in die Benutzung der neufranzösischen Wörterbücher, Tübingen, Max Niemeyer.

HAUSMANN, Franz Josef (1979) : «Un dictionnaire des collocations est-il possible?», Travaux de Linguistique et de Littérature XVII, 1, pp. 187-195.

HAUSMANN, Franz Josef (1985): "Kollokationen im deutschen Wörterbuch. Ein Beitrag zur Theorie des lexikographischen Beispiels," Bergenholtz/Mugdan (Eds.), pp. 118-129.

HAUSMANN, Franz Josef (1988): "Grundprobleme der zweisprachigen Lexikographie", HyldgaardJensen/Zettersten (Ed.), Tübingen, Max Niemeyer.

HVALKOF, Sonja (1985): Étude comparative des données terminologiques des banques de terminologie Danterm, B.T.Q.. Eurodicautom, Normaterm, O.F.L. et Siemens, 1-2, Kobenhavn, Handelshojskolen.

HYLDGAARD-JENSEN Karl and Arne ZETTERSTEN (Eds.)(1988): Symposium on Lexicography, 3, Proceedings, Tübingen, Max Niemeyer.

KITTREDGE, Richard; Polguere, Alain and L. IORDANSKAJA (1988): "Multilingual Text Generation and the Meaning-Text Theory," Second International Conference on Theoretical and Methodological Issues in Machine Translation of Natural Languages, Carnegie Mellon University, Pittsburgh, Pennsylvania, June 1214 , Proceedings.

KNOWLES, Francis E. (1989): "The Role of the 'Learner's Dictionary' in the Training of Translators: Some Observations on the Soviet Approach", Snell-Hornby/Pochl (Eds.), pp. 139-148.

KROMANN, Hans-Peder; RIIBER, Theis and Poul ROSBACH (1984): "Active and Passive Bilingual Dictionaries: The Scerba Concept Reconsidered", Proceedings of Lexeter-83, Tübingen, Max Niemeyer, pp. $207-215$. 
KROMANN, Hans-Peder, RIIBER, Theis and Poul ROSBACH (1989): "Principles of bilingual lexicography", à paraître dans Hausmann, Franz-Josef/Reichmann, Oskar/Wiegand, Herbert-Ernst/Zgusta, Ladislav (Eds.), Dictionaries, Dictionnaires, Wörterbücher, Ein internationales Handbuch, Berlin, de Gruyter.

KROMANN, Hans-Peder (1989): "Neue Orientierung der zweisprachigen Wörter-bücher. Zur funktionalen zweisprachigen Lexikographie", Snell-Hornby/Poehl (Eds.), pp. 55-65.

LACROIX, Ulysse (1931): Les mots et les idées, Paris, Nathan.

LEROT, Jacques and Rudolf KERN (Eds.)(1978): Mélanges de littérature et de linguistique, offerts au professeur Henri Draye à l'occasion de son éméritat, Louvain.

MAYER, Renate and Elisabeth MAIER: "Spezifikation eines konzeptionellen Schemas für Terminologiedatenbanken", Czap/Galinski, pp. 151-163.

MELC̆UK, Igor A. (avec ARBATCHEWSKY-JUMARIE, Nadja/ELNITSKY, Léo/ IORDANSKAJA, Lidija/LESSARD, Adèle)(1984): Dictionnaire explicatif et combinatoire du français contemporain, Recherches lexico-sémantiques, Montréal, Les Presses de l'Université de Montréal.

MELCUK, Igor A. et al. (1989): Dictionnaire explicatif et combinatoire du français contemporain, Recherches lexico-sémantiques, Montréal, Les Presses de l'Université de Montréal.

MELČCUK, Igor, and Alain POLGUERE (1987): "A Formal Lexicon in the Meaning-Text Theory (or how to do Lexica with Words)," Computational Linguistics 13, 3-4, pp. 261-275.

PICHT, Heribert (1987): "Terms and their LSP environment - LSP Phraseology", Meta, 'Special Issue on Terminotics'.

PICHT, Heribert (1987): "Fachsprachliche Phraseologie - Die terminologische Funktion von Verben", Czap/Galinski, pp. 21-34.

REUM, Albrecht et Louis CHAMBILLE (1911): Guide-lexique de composition française, Leipzig, Bibliographisches Institut.

ROEY, Jacques van (1978): "Collocation in lexical analysis," Lerot/Kern, pp. 155-162.

ROOS, Eckhard (1975): Kollokationsmöglichkeiten der Verben des Sehvermögens im Deutschen und Englischen, Bern/ Frankfurt/M. Lang.

ROSSENBECK, Klaus (1989); "Lexikologische und lexikographische Probleme fachsprachlicher Phraseologie aus kontrastiver Sicht", Snell-Hornby/Poehl (Eds.), pp. 197-210.

SCHAETZEN, Caroline de (Ed.)(1989): Terminologie diachronique, Paris, Conseil international de la langue française.

SINCLAIR, John McH. (1987): “Collocation. A progress report," Steele/Threadgold (Eds.), pp.319-331.

SNELL-HORNBY, Mary and Esther PÖHL (Eds.)(1989): Translation and Lexicography, Amsterdam, Benjamins, Paintbrush Special Monograph.

STEELE, Ross and Terry THREADGOLD (Eds.)(1987): Language Topics. Essays in Honour of Michael Halliday, Amsterdam/Philadelphia, Benjamins.

STEINER, Erich (1983): Die Entwicklung des Britischen Kontextualismus, Heidelberg, Groos.

VIEHWEGER, Dieter (1982): "Die Darstellung semantischer Vereinbarkeits-beziehungen zwischen lexikalischen Elementen im einsprachigen Wörterbuch des Deutschen," Agricola, Erhard/ Schildt, Jochen/ Viehweger, Dieter (Eds.), Wortschatzforschung heute. Aktuelle Probleme der Lexikologie und Lexikographie, Leipzig, Enzyklopädie, pp. 23-41.

WAGNER, Horst (1986): «Les dictionnaires du français langue de spécialité/langue économique», XVIII Congrès international de linguistique et philologie romanes, Trèves 1986, Proceedings. 